07

\title{
Методы переключения поляризации излучения в GaAs лазерных диодах
}

\author{
() М.В. Дорохин, Б.Н. Звонков, П.Б. Демина, Н.В. Дикарева, А.В. Здоровейщев, А.В. Кудрин, \\ О.В. Вихрова, И.В. Самарцев, С.М. Некоркин
}

Нижегородский государственный университет им. Н.И. Лобачевского, 603950 Нижний Новгород, Россия

e-mail: woterbox@mail.ru

Поступило в Редакцию 16 февраля 2021 г.

В окончательной редакции 22 апреля 2021 г.

Принято к публикации 28 апреля 2021 г.

Приведены результаты исследования режимов работы лазерных структур с управляемой поляризацией света, в том числе результаты измерения поляризационных характеристик. Показана возможность управления как линейной, так и циркулярной поляризацией света при соответствующей модификации конструкции лазера. В частности, осуществлена устойчивая лазерная генерация на двух ортогонально-поляризованных модах в ближнем ИК диапазоне с соотношением интенсивностей компонент излучения $I_{\mathrm{TE}} / I_{\mathrm{TM}} \approx 4.5$. Реализована возможность генерации циркулярно-поляризованного излучения в торцевых лазерных диодах посредством намагничивания комбинированного полупрозрачного зеркала с ферромагнитным слоем CoPt, нанесенного на торец лазерного резонатора. В режиме насыщения намагниченности слоя СоРt значение степени поляризации составило $\pm 1.25 \%$.

Ключевые слова: полупроводники, лазерный диод, квантовая яма, поляризация.

DOI: 10.21883/JTF.2021.09.51221.39-21

\section{Введение}

Управление поляризацией лазерного излучения одна из важнейших практических задач целого ряда современных научных направлений: радиофотоники, оптоэлектроники, спинтроники [1-3]. Источники света с управляемыми интенсивностью и поляризацией могут выполнять роль логической ячейки, интегрированной в большую матрицу, в которой к „стандартному“ каналу кодирования информации бинарным сигналом высокой и низкой интенсивности добавляется дополнительный канал кодирования информации за счет поляризации света (двух взаимно перпендикулярных направлений линейной поляризации, либо знака циркулярной поляризации). Это позволит повысить производительность оптоэлектронных схем как минимум в два раза [1].

„Стандартные“ методы создания поляризации, основанные на преобразовании света с помощью электрооптических кристаллов, уже не удовлетворяют требованиям компактности и интегрируемости в оптоэлектронную схему. Одним из новых принципов управления поляризационными свойствами излучения является использование анизотропии преломления оптического кристалла за счет приложения внешнего воздействия [4,5]. Приложение внешнего воздействия (механическое сжатие) позволяет понизить степень симметрии лазерного кристалла, благодаря чему в кристаллах кубической сингонии (в том числе $\mathrm{A}^{3} \mathrm{~B}^{5}$ ) становится возможным существование двух взаимно перпендикулярных направлений поляризации (явление двулучепреломления). Первые результаты по изучению данного явления в струк- туре гетеролазеров были приведены в работах [6,7]. Недостатком этого принципа является необходимость механического воздействия на структуру, что сложно реализуемо технически, кроме того, механическое воздействие повышает вероятность износа. Известным в настоящее время способом создания когерентных излучателей с циркулярной поляризацией является применение электрической инжекции спин-поляризованных носителей из подходящего ферромагнитного контакта или разбавленного магнитного полупроводника $[8,9]$. Основными конструкциями таких излучателей являются дорогостоящие поверхностно- излучающие лазеры с вертикальным резонатором (VCSEL) [10], лазеры с массивом квантовых точек в активной области, либо GaN-лазеры [11].

Другим новым подходом к управлению поляризацией света, лишенным недостатков, перечисленных выше, являются схемы полупроводниковых лазеров, в которых возможность управления реализована конструктивно. В частности, в настоящей работе рассмотрены бистабильные лазеры с управляемой электрическим полем линейной поляризацией, а также лазеры, генерирующие циркулярно-поляризованное излучение.

Бистабильные лазерные диоды полосковой геометрии, работающие в ближнем ИК диапазоне, представляют собой гетероструктуру с двумя квантовыми ямами (КЯ), генерирующими линейно-поляризованное излучение с взаимно-перпендикулярными плоскостями поляризации. Для создания лазеров, испускающих свет круговой поляризации, использован новый подход, основанный на формировании функционального слоя ла- 
зерной структуры (зеркала лазерного резонатора) из ферромагнитного материала. Спин-зависимое отражение или пропускание лазерного излучения ферромагнитным зеркалом обусловливает появление в излучении циркулярно-поляризованной компоненты [12]. В работе приводятся результаты исследования режимов работы лазерных структур с управляемой поляризацией света, в том числе результаты измерения поляризационных характеристик. Показана возможность управления как линейной, так и циркулярной поляризацией света при соответствующей модификации конструкции лазера.

\section{1. Методика эксперимента}

В настоящей работе были разработаны и экспериментально исследованы два типа полупроводниковых лазеров. Первый тип - бистабильный лазер, генерирующий излучение на двух близких частотах с разными линейными взаимно ортогональными поляризациями (ТЕ- и ТМмоды). Второй тип - лазер, генерирующий частично циркулярно-поляризованное излучение за счет магнитооптических эффектов, возникающих при прохождении и отражении света в ферромагнитном покрытии, являющимся элементом полупрозрачного зеркала лазерного резонатора.

Все исследуемые лазерные структуры выращивались методом МОС-гидридной эпитаксии в горизонтальном кварцевом реакторе. Гетероструктура (ГС) лазеров 1-го типа содержала следующие слои: буферный слой $n$-GaAs $(500 \mathrm{~nm})$, градиентный ограничительный слой $n$-AlGaAs $(100 \mathrm{~nm})$, ограничительный слой $n$ $\mathrm{Al}_{0.65} \mathrm{Ga}_{0.35} \mathrm{As}(1090 \mathrm{~nm})$, волноводный слой $\mathrm{Al}_{0.4} \mathrm{Ga}_{0.6} \mathrm{As}$ $(350 \mathrm{~nm})$, КЯ1 - AlInGaAs $(10 \mathrm{~nm})$, волноводный слой $\mathrm{Al}_{0.4} \mathrm{Ga}_{0.6} \mathrm{As}(50 \mathrm{~nm})$, КЯ2 - GaAsP $(10 \mathrm{~nm})$, волноводный слой $\mathrm{Al}_{0.4} \mathrm{Ga}_{0.6} \mathrm{As}(350 \mathrm{~nm})$, ограничительный слой $p-\mathrm{Al}_{0.65} \mathrm{Ga}_{0.35} \mathrm{As}(1090 \mathrm{~nm})$, градиентный ограничительный слой $p$-AlGaAs $(100 \mathrm{~nm})$, контактный слой $p^{+}$-GaAs $(300 \mathrm{~nm})$.

Тип верхней дырочной подзоны квантовой ямы, согласно правилам отбора [13], определяет вид поляризации лазерной моды, которая генерируется в этой квантовой яме. В КЯ, использованных в настоящей работе, анизотропная деформация, вызванная действием упругих напряжений растяжения и сжатия, приводит к изменениям симметрии кристаллической решетки. Это позволяет снять вырождение валентной зоны в Г-точке, в результате чего между подзонами „тяжелых“ и ,легких“ дырок появляется энергетическая щель, и происходит перераспределение пространственного расположения уровней „легких“ и „тяжелых“ дырок в КЯ, являющихся активной средой лазера. В результате модифицируются излучательные характеристики прибора. Так, например, известно, что одноосное сжатие по нормали к плоскости структуры ведет к переходу в основное состояние в квантовой яме уровня „легких“ дырок LH1, тогда как в биаксиально растянутой КЯ основным состоянием валентной зоны является уровень „тяжелых“ дырок HН1 [14,15]. Излучательная рекомбинация с участием оптического перехода свободный электрон-,легкая“ дырка сопровождается испусканием ТМ-линейно-поляризованного света, для которого вектор $\mathbf{E}$ электрического поля электромагнитной волны расположен перпендикулярно плоскости квантовой ямы. Излучательная рекомбинация с участием оптического перехода свободный электрон-,тяжелая“ дырка сопровождается испусканием ТЕ-поляризованого света, в котором вектор электрического поля расположен в плоскости КЯ. Здесь и далее плоскостью поляризации света будем считать плоскость поляризации вектора $\mathbf{E}$ электромагнитной волны.

В рассматриваемых экспериментальных лазерных структурах состав квантовых ям подбирался таким образом, чтобы за счет рассогласования параметров решетки одна из них испытывала деформации растяжения (GaAsP), а вторая - сжатия (AlInGaAs). Таким образом, формировалась активная область со знакопеременными упругими напряжениями, способная к генерации как ТЕ-, так и ТМ-поляризованной моды.

Гетероструктура лазеров 2-го типа (предназначенная для генерации циркулярно-поляризованного излучения) содержала следующие слои: буферный слой $n$ GaAs $(380 \mathrm{~nm})$, ограничительный слой $n$ - $\mathrm{In}_{0.49} \mathrm{Ga}_{0.51} \mathrm{P}$ $(840 \mathrm{~nm})$, волноводный слой InGaAsP $(590 \mathrm{~nm})$, волноводный слой GaAs $(30 \mathrm{~nm})$, КЯ - InGaAs $(10 \mathrm{~nm})$, волноводный слой InGaAsP $(420 \mathrm{~nm})$, волноводный ограничительный слой $p-\mathrm{In}_{0.49} \mathrm{Ga}_{0.51} \mathrm{P}(840 \mathrm{~nm})$, контактный слой $p^{+}$-GaAs $(250 \mathrm{~nm})$. Содержание индия в составе КЯ составляло $x=0.18$.

На основе сформированных гетероструктур были изготовлены лазерные диоды полоскового типа с шириной полоска $100 \mu \mathrm{m}$ (рис. 1). Изготовление осуществлялось методом фотолитографии и прецизионного химического травления с последующей протонной имплантацией областей, расположенных вне контактного полоска [16].

Зеркалами резонаторов в лазерных диодах 1-го типа служили сколотые грани структуры, а в диодах 2-го типа - отражающее диэлектрическое покрытие и комбинированное покрытие, представляющее собой последовательно сформированные просветляющее диэлектрическое покрытие и тонкий слой ферромагнитного металла $(8 \mathrm{~nm} \mathrm{CoPt})$. Формирование и управление циркулярнополяризованным излучением в диодах 2-го типа осуществлялось путем перемагничивания CoPt-слоя. Малая толщина данного слоя позволяет свести к минимуму его возможный разогрев и деградацию при мощности падающего лазерного излучения $\sim 500 \mathrm{~mW}$, а просветляющее покрытие на основе диэлектрика обеспечивает электрическую изоляцию слоя СoPt от остальных слоев структуры и исключает его влияние на электрические характеристики диода. Схема образцов для исследования представлена на рис. 1. Для диодов 2-го типа была также сформирована контрольная структура, не содержащая слоя CoPt. 


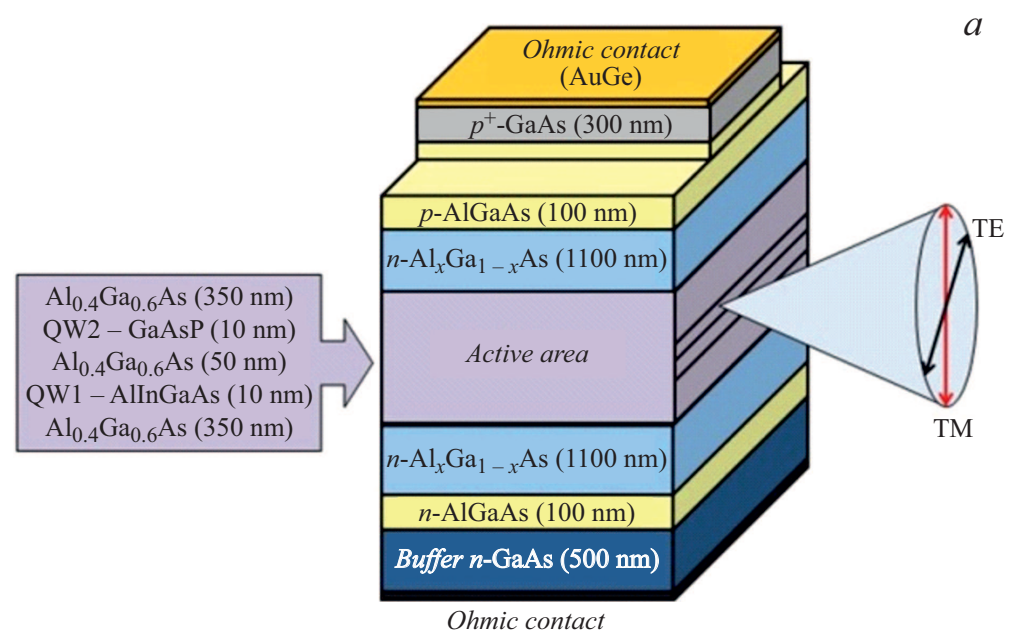

Рис. 1. $a-$ схема лазерного диода 1-го типа с управляемой линейно поляризацией. Стрелкой отмечено направление вектора $E$ для ТЕ- и ТМ-мод; $b$ - схема лазерного диода 2-го типа с управляемой циркулярной поляризацией. Вид поляризации (правая, левая) зависит от намагниченности слоя CoPt.

Исследования излучательных свойств образцов гетеролазеров проводились при температуре $300 \mathrm{~K}$ в непрерывном и импульсном режимах накачки. Предметом исследований являются спектральные зависимости, а также поляризация излучения лазерных диодов. Измерение линейной поляризации проводилось с использованием линейного поляризатора (призмы Глана), монохроматора МДР-23 и детектора. Измерение циркулярной поляризации лазерного излучения проводились по стандартной методике с использованием $\lambda / 4$-пластины, призмы Глана, монохроматора и детектора ([11]). Для получения циркулярно-поляризованного света образцы вводились во внешнее магнитное поле величиной до $300 \mathrm{mT}$, направленное вдоль лазерного волновода (и перпендикулярно полупрозрачному зеркалу с ферромагнитным слоем $\mathrm{CoPt}($ рис. $1, b))$.

Значение степени циркулярной поляризации определялось по относительной интенсивности лево- и правополяризованной компонент излучения в соответствии с формулой (1):

$$
P_{\mathrm{EL}}=\left(I^{+}-I^{-}\right) /\left(I^{+}+I^{-}\right) \cdot 100 \%,
$$

где $I^{+}$и $I^{-}-$интенсивность излучения, поляризованного по левому и по правому кругу соответственно.

На контрольных структурах, представляющих собой пленки CoPt, аналогичные по составу и толщине исследованным и сформированные на пластинах полуизолирующего GaAs, были выполнены измерения магнитополевой зависимости намагниченности в диапазоне напряженности внешнего магнитного поля $\pm 170 \mathrm{mT}$. Измерения выполнялись при комнатной температуре с использованием магнетометра с переменным градиентом поля.

\section{2. Результаты и обсуждение}

Спектры электролюминесценции лазерных диодов 1-го типа, измеренные с использованием линейного поляризатора, приведены на рис. 2. Измерения проводились при непрерывном режиме накачки электрическим током.

Спектр электролюминесценции в допороговом режиме представлен на рис. 2, a (кривая 1). Спектр представляет собой сумму двух компонент с близкими частотами и взаимно перпендикулярными плоскостями линейной поляризации (кривые 2, 3). Измерения с использованием поляризатора показали, что пик электролюминесценции с длиной волны в максимуме, равной $806 \mathrm{~nm}$, соответствует ТМ-моде (кривая 2), а с длиной волны $809 \mathrm{~nm}-$ ТЕ-моде (кривая 3). Пороговый ток лазерной генерации для указанной структуры составил $(500 \pm 20) \mathrm{mA}$. С увеличением тока накачки до $1500 \mathrm{~mA}$ (значительно выше порога генерации) для обеих линейно-поляризованных компонент (мод) наблюдается сдвиг максимумов интенсивности в длинноволновую область, а разница между положением этих максимумов составляет $(1.5 \pm 0.5) \mathrm{nm}$. Длина волны максимума излучения, поляризованного в плоскости КЯ (ТМ-мода), составила $\sim 817 \mathrm{~nm}$, для поляризованной перпендикулярно ТЕ-моды длина волны $\sim 818 \mathrm{~nm}$ (рис. $2, b$ ). Таким образом, для данных условий лазерной генерации диода 1-го типа общий спектр излучения представляет собой суперпозицию двух компонент с взаимно перпендикулярной поляризацией, а соотношение интенсивностей этих компонент составляет $I_{\mathrm{TE}} / I_{\mathrm{TM}} \approx 4.5$.

На рис. 3 представлена зависимость соотношения интенсивностей $I_{\mathrm{TE}} / I_{\mathrm{TM}}$ от величины рабочего тока. Во всем исследованном диапазоне токов это соотношение отлично от нуля, т. е. имеет место двухполосная генерация на ортогонально-поляризованных модах, а наиболь- 

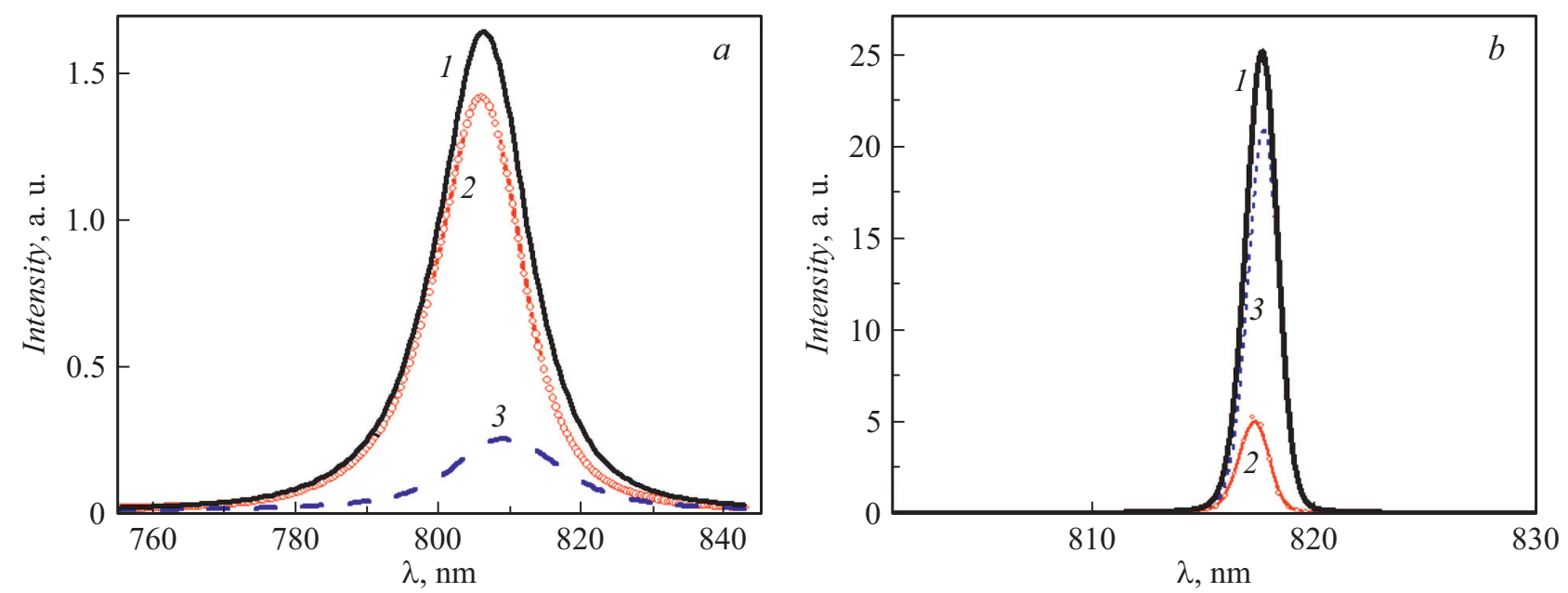

Рис. 2. Спектры электролюминесценции лазерного диода 1: $a-$ величина тока $400 \mathrm{~mA}$ (допороговый режим), $b-$ величина тока $1500 \mathrm{~mA}$ (послепороговый режим); 1 - суммарный спектр ЭЛ; 2 - спектр компоненты излучения, поляризованной перпендикулярно плоскости КЯ (ТМ-мода), 3 - спектр компоненты излучения, поляризованной в плоскости КЯ (ТЕ-мода).

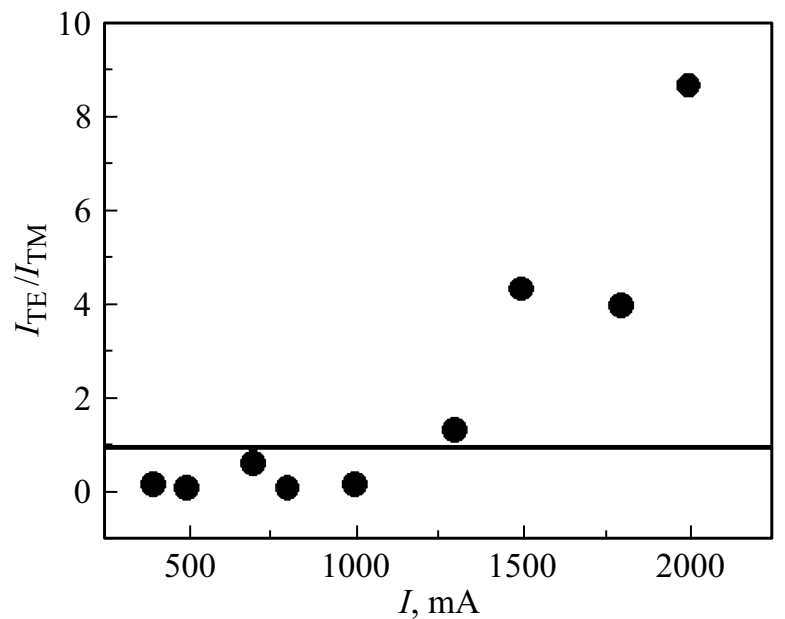

Рис. 3. Зависимость соотношения интенсивностей взаимно перпендикулярных компонент линейно-поляризованного лазерного излучения от величины тока диода. Линией отмечен уровень $I_{\mathrm{TE}} / I_{\mathrm{TM}}=1$.

шее значение квантовой эффективности достигает $30 \%$ даже в отсутствие просветляющих и отражающих диэлектрических покрытий граней лазерного резонатора. Соотношение интенсивностей компонент линейной поляризации зависит от тока накачки. При низких значениях тока доминирует ТМ-компонента поляризации, т.е. значение $I_{\mathrm{TE}} / I_{\mathrm{TM}}$ меньше единицы (уровень $I_{\mathrm{TE}} / I_{\mathrm{TM}}=1$ отмечен линией на графике). Наименьшее зарегистрированное значение $I_{\mathrm{TE}} / I_{\mathrm{TM}}$ составило 0.11 , т.е. интенсивность ТМ-компоненты излучения примерно на порядок выше, чем для противоположной. При постепенном увеличении тока накачки выше порогового до $1000 \mathrm{~mA}$ наблюдается пропорциональный рост интенсивностей как ТМ-, так и ТЕ-моды, так что их соотношение остается в пределах погрешности измерений величиной постоянной. При величине рабочего тока выше $1000 \mathrm{~mA}$ происходит перераспределение интенсивностей между компонентами, и значение $I_{\mathrm{TE}} / I_{\mathrm{TM}}$ становится больше единицы, т.е. начинает преобладать ТЕ-компонента лазерного излучения. В области более $1200 \mathrm{~mA}$ рост интенсивности ТЕ-моды идет одновременно с ростом интенсивности ТМ-моды, но скорость роста интенсивности ТЕ-моды в данном диапазоне токовой накачки превышает скорость роста интенсивности ТМмоды. Наблюдаемая картина предположительно может быть связана с двумя обстоятельствами:

- за порогом лазерной генерации основной вклад в поляризацию излучения вносят оптические свойства резонатора (коэффициент отражения на гранях резонатора Фабри-Перо, как правило, приводит к выбору ТЕполяризации);

- наличием большего числа уровней „тяжелых“] дырок в квантовой яме AlInGaAs/GaAs, а также в квантовой яме $\mathrm{GaAsP} / \mathrm{GaAs}$ вблизи уровня континуума, по сравнению с количеством уровней „легких“ дырок вблизи потолка валентной зоны.

Последнее обстоятельство может объяснить отсутствие области насыщения на графике зависимости соотношения интенсивностей взаимно перпендикулярных компонент линейно-поляризованного лазерного излучения от величины тока накачки.

Наблюдаемое различие в интенсивностях генерации при токах накачки 1500 и $1800 \mathrm{~mA}$, вероятно, обусловлено возможностью перемешивания уровней „тяжелых“ и „легких“ дырок в области возбужденных состояний как в упруго-растянутой, так и в сжатой КЯ, и/или потерей устойчивости поперечного распределения поля волноводной моды из-за пространственно неоднородного выгорания носителей заряда. 


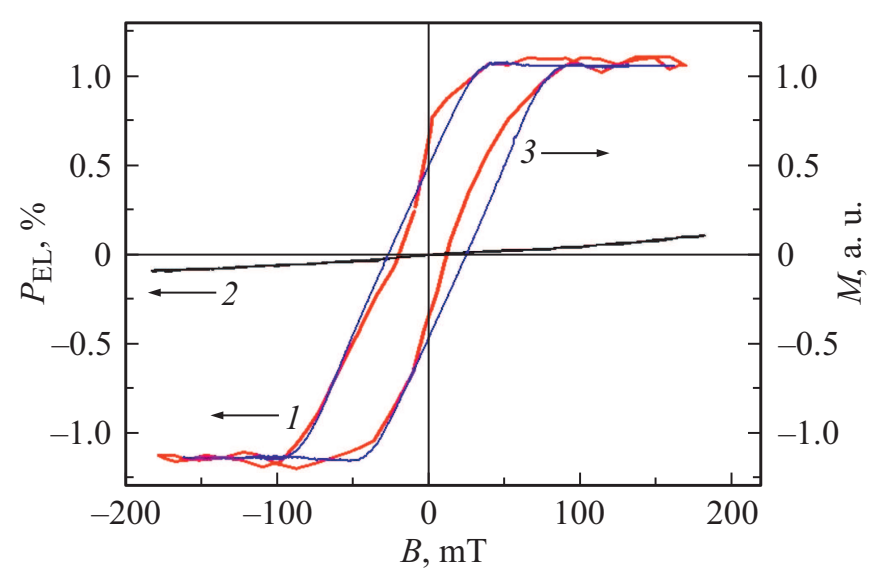

Рис. 4. Магнитополевая зависимость степени циркулярной поляризации излучения (слева) лазерного диода с покрытием $\mathrm{CoPt}$ (кривая 1), лазерного диода без покрытия CoPt (кривая 2) и намагниченности СoPt слоя (справа, кривая 3).

При максимальном значении тока $(2000 \mathrm{~mA})$ соотношение интенсивностей составляет 9 , т.е. интенсивность ТЕ-компоненты излучения почти на порядок выше, чем ТМ-компоненты. Таким образом, изменяя величину тока накачки, можно управлять величиной вклада в общую интенсивность излучения лазера отдельной линейнополяризованной моды.

Следует подчеркнуть, что линии лазерной генерации двух ортогонально-поляризованных компонент излучения (мод) имеют максимально близкие длины волн, что говорит о наличии минимального энергетического интервала между уровнями размерного квантования „легких“ и „тяжелых“ дырок в валентной зоне активной среды лазера 1-го типа. Поэтому вероятность излучательного перехода свободный электрон-LH1 для растянутой КЯ $(\mathrm{GaAs} / \mathrm{GaAs})$ сопоставима с вероятностью излучательного перехода свободный электрон-НH1 для сжатой КЯ (AlInGaAs/GaAs), что, в свою очередь, обусловливает „переключение“ между компонентами линейной поляризации при изменении условий накачки (рис. 3).

Рассмотрим работу лазерного диода 2, сконструированного для генерации частично циркулярнополяризованного света. Для данного вида структур не обсуждается работа активной области гетеролазера, а управление циркулярной поляризацией лазерного излучения осуществляется путем перемагничивания слоя $\mathrm{CoPt}$, нанесенного поверх выходного полупрозрачного зеркала. На рис. 4 представлена зависимость степени циркулярной поляризации лазерного излучения диода 2 от внешнего магнитного поля, направленного вдоль плоскости квантовой ямы и перпендикулярно плоскости слоя $\mathrm{CoPt}$ (направление магнитного поля показано на рис. $1, b)$. Полученная кривая описывает петлю гистерезиса со значением магнитного поля насыщения вблизи $100 \mathrm{mT}$. Отметим, что магнитополевая зависимость степени циркулярной поляризации излучения подобна магнитополевой зависимости намагниченности пленки $\mathrm{CoPt}$, осажденной на контрольную структуру с теми же параметрами нанесения. При насыщении намагниченности слоя CoPt значение степени поляризации составило $\pm 1.25 \%$. Значение поляризации в нулевом магнитном поле составило $\approx 0.5 \%$.

Тонкие пленки $\mathrm{CoPt}$, полученные методом электронно-лучевого испарения в вакууме, характеризуются перпендикулярным слою направлением оси легкого намагничивания, коэрцитивными полями до $200 \mathrm{mT}$ (в зависимости от состава пленки) и способностью оставаться намагниченными в отсутствие внешнего магнитного поля при комнатной температуре [17]. Помимо этого, для указанных пленок характерны аномально высокие характеристики эффекта магнитно-кругового дихроизма (МКД), заключающегося в циркулярной поляризации прошедшего света [18]. По данным [18] для излучения в диапазоне длин волн 950-980 nm максимальная степень циркулярной поляризации света, прошедшего через пленку CoPt с параметрами, аналогичными использованным в настоящей работе, составляет $0.5 \%$.

Подобие магнитополевых зависимостей намагниченности и степени циркулярной поляризации лазерного излучения (рис. 4 кривые 1 и 3), а также сопоставление поляризационных свойств излучения лазера с наличием тонкого слоя ферромагнитного металла $\mathrm{CoPt}$ (рис. 4, кривая 1) на поверхности просветляющего диэлектрического покрытия и без пленки $\mathrm{CoPt}$ (рис. 4, кривая 2), позволяет заключить, что зарегистрированная нами циркулярная поляризация лазерного излучения связана с магнитооптическими эффектами, протекающими при отражении и пропускании света через слой $\mathrm{CoPt}$, а именно аддитивным действием двух эффектов: спинзависимого отражения стимулированного излучения от полупрозрачного зеркала $\mathrm{CoPt}$ и магнитно-кругового дихроизма, заключающегося в поляризации излучения, прошедшего через намагниченный слой $\mathrm{CoPt}[12,18]$. Эффект магнитно-кругового дихроизма дает вклад в циркулярную поляризацию света на уровне $0.5 \%$ [18]. Помимо этого, вклад в итоговое значение степени циркулярной поляризации, по-видимому, дает поляризация света, возникающая при отражении от намагниченного CoPt. Степень циркулярной поляризации света при однократном отражении от намагниченного $\mathrm{CoPt}$, согласно [19], может принимать значение около $0.16 \%$. В случае лазерных диодов излучение перед выходом через полупрозрачное зеркало испытывает многократное отражение, в результате чего итоговое значение степени циркулярной поляризации может быть повышено. Дальнейшее повышение степени циркулярной поляризации относительно значения, полученного в настоящей работе, возможно посредством оптимизации лазерного волновода и толщин полупрозрачного зеркала. 


\section{Заключение}

В работе показана возможность управления поляризацией излучения полупроводникового лазера посредством формирования специализированных лазерных структур, имеющих определенные конструкционные особенности. Управление линейной поляризацией света (переключение между ТМ- и ТЕ-модами лазерного излучения) осуществляется величиной тока накачки лазерного диода, содержащего в активной области две квантовые ямы, подверженные деформациям растяжения и сжатия. Управление циркулярной поляризацией осуществляется за счет намагничивания комбинированного полупрозрачного зеркала с ферромагнитным слоем CoPt, нанесенного на торец лазерного резонатора „стандартного“ полупроводникового полоскового лазерного диода. Полученные эффекты представляются перспективными для создания дополнительных каналов передачи информации в схемах радиофотоники.

\section{Благодарности}

Авторы выражают благодарность в подготовке эксперимента А.А. Бирюкову и М.В. Карзановой, а также И.Л. Калентьевой за помощь в подготовке работы.

\section{Финансирование работы}

Работа выполнена при финансовой поддержке Программы повышения конкурентоспособности 5-100.

\section{Конфликт интересов}

Авторы заявляют, что у них нет конфликта интересов.

\section{Список литературы}

[1] A. Hirohata, K. Yamada, Y. Nakatanietal. J. Magnetism. Mag. Mater., 509, 166711 (2020). DOI: $10.1016 /$ j.jmmm.2020.166711

[2] V. Torres-Company, A.W. Weiner. Laser Photon. Rev., 8 (3), 368 (2014). DOI: 10.1002/lpor.201300126

[3] R.I. Sabitu, N.G. Khan, A. Malekmohammadi. Opto-Electron. Rev., 27 (3), 252 (2019). DOI: 10.1016/j.opelre.2019.07.001

[4] E.V. Bogdanov, H. Kissel, K.I. Kolokolov, N.Ya. Minina. Semicond. Sci. Technol., 31 (3), 035008 (2016). DOI: $10.1088 / 0268-1242 / 31 / 3 / 035008$

[5] E.V. Bogdanov, K.I. Kolokolov, N.V. Melnikova, N.Ya. Minina, G.V. Tikhomirova. IOP Conf. Series: J. Phys., 950 (4), 042047 (2017). DOI: 10.1088/1742-6596/950/4/042047

[6] D. Sun, D.P. Bour, K.J. Beernink, D.W. Treat, R.D. Bringans. SPIE, 2682, 108 (1996). DOI: 10.1117/12.237646

[7] В.Я. Алешкин, А.А. Дубинов. Квант. электрон., 39 (8) (2009). [V.Ya. Aleshkin, A.A. Dubinov. Quantum Electron., 39 (8), 727 (2009). DOI: 10.1070/QE2009v039n08ABEH014087]

[8] I. Zutic, J. Fabian, S. Das Sarma. Rev. Mod. Phys., 76 (2), 323 (2004). DOI: 10.1103/RevModPhys.76.323
[9] S.H. Liang, T.T. Zhang, P. Barate, J. Frougier, M. Vidal, P. Renucci, B. Xu, H. Jaffrés, J.-M. George, X. Devaux, M. Hehn, X. Marie, S. Mangin, H.X. Yang, A. Hallal, M. Chshiev, T. Amand, H.F. Liu, D.P. Liu, X.F. Han, Z.G. Wang, Y. Lu. Phys. Rev. B, 90 (8), 085310 (2014). DOI: 10.1103/PhysRevB.90.085310

[10] K. Ikeda, T. Fujimoto, H. Fujino, T. Katayama, S. Koh, H. Kawaguchi. IEEE Photonics Technology Letters, 21 (18), 1350 (2009). DOI: 10.1109/LPT.2009.2026631

[11] M. Holub, P. Bhattacharya. J. Phys. D: Appl. Phys., 40 (11), R179 (2007). DOI: 10.1088/0022-3727/40/11/R01

[12] А.В. Кудрин, М.В. Дорохин, А.В. Здоровейщев, Б.П. Демина, О.В. Вихрова, И.Л. Калентьева, М.В. Ведь. ФТТ, 59 (11), 2203 (2017). DOI: 10.21883/FTT.2017.11.45062.17k [A.V. Kudrin, M.V. Dorokhin, A.V. Zdoroveishchev, P.B. Demina, O.V. Vikhrova, I.L. Kalent'eva, M.V. Ved'. Phys. Solid State, 59 (11), 2223 (2017). DOI: $10.1134 / \mathrm{S} 106378341711018 \mathrm{X}$

[13] F. Bachmann, P. Loosen, R. Poprawe. Springer-Verlag, NY., 128, 554 (2007).

[14] П.Г. Елисеев, Б. Н. Свердлов, Н. Шохуджаев. Квант. электрон., 11 (8), 1665 (1984). [P.G. Eliseev, et al. Soviet J. Quant. Electron., 14 (8), 1120 (1984). DOI: 10.1070/QE1984v014n08ABEH005386]

[15] Е.В. Богданов, Н.Б. Брандт, Н.Я. Минина, С.С. Широков. ВМУ. Сер. 3. Физика. Астрономия, 2-11 (6), 74 (2011).

[16] P. deSouza, I. Danilov, H. Boudinov. Appl. Phys. Lett., 68 (4), 535 (1996). DOI: 10.1063/1.116391

[17] А.В. Здоровейщев, О.В. Вихрова, П.Б. Демина, М.В. Дорохин, А.В. Кудрин, А.Г. Темирязев, М.П. Темирязева. ФТТ, 61 (9), 1628 (2019). DOI: 10.21883/FTT.2019.09.48101.04N [A.V. Zdoroveyshchev, O.V. Vikhrova, P.B. Demina, M.V. Dorokhin, A.V. Kudrin, A.G. Temiryazev, M.P. Temiryazeva. Phys. Solid State, 61, 1577 (2019). DOI: $10.1134 / \mathrm{S} 1063783419090294]$

[18] А.В. Кудрин, А.В. Здоровейщев, О.В. Вихрова, М.В. Дорохин, И.Л. Калентьева, П.Б. Демина. ФТТ, 60 (11), 2236 (2018). DOI: 10.21883/FTT.2018.11.46669.11NN [A.V. Kudrin, A.V. Zdoroveyshchev, O.V. Vikhrova, M.V. Dorokhin, I.L. Kalent'eva, P.B. Demina. Phys. Solid State, 60, 2276 (2018). DOI: 10.1134/S1063783418110161]

[19] М.В. Дорохин, П.Б. Демина, А.В. Буданов, Ю.Н. Власов, Г.И. Котов, А.В. Здоровейщев, В.Н. Трушин, Б.Н. Звонков. Письма в ЖТФ, 45|,(5), 52 (2019). DOI: 10.21883/PJTF.2019.05.47400.17588 [M.V. Dorokhin, P.B. Demina, A.V. Bulanov, Yu.N. Vlasov, G.I. Kotov, A.V. Zdoroveyshchev, V.N. Trushin, B.N. Zvonkov. Tech. Phys. Lett., 45, 235 (2019). DOI: 10.1134/S1063785019030064] 